\title{
Patients' Perspectives and Needs on Novel Food Allergy Treatments in the United States
}

Linda Herbert, $P h D^{1,2, *}$

Mary Jane Marchisotto, MBA ${ }^{3,4}$

Brian Vickery, $M D^{5}$

\section{Address}

${ }^{*}, 1$ Children's National Hospital, 111 Michigan Ave, NW, Washington, DC 20010,

USA

Email: therbert@childrensnational.org

${ }^{2}$ George Washington University School of Medicine, Washington, DC USA

${ }^{3}$ MJM Advisory, New York, NY USA

${ }^{4}$ Mary H. Weiser Food Allergy Center, University of Michigan Medicine, Ann Arbor, MI USA

${ }^{5}$ Emory University, Atlanta, GA USA

Published online: 23 January 2021

(C) The Author(s), under exclusive licence to Springer Nature Switzerland AG part of Springer Nature 2021

This article is part of the Topical Collection on Food Allergy

Keywords Food allergy $\cdot$ Treatment · Patients · Quality of life

Abbreviations EPIT Epicutaneous immunotherapy - ICER Institute for Clinical and Economic Review - FAQLQ Food Allergy Quality of Life Questionnaires . FARE Food Allergy Research \& Education . FDA Food and Drug Administration - PRO Patient-reported outcomes - QoL Quality of life - SLIT Sublingual immunotherapy • US United States

\section{Abstract}

Purpose Food allergy management places a daily psychosocial burden on patients and their caregivers. New food allergy treatments may positively impact their lives, but also introduce new stressors. The purpose of this paper is to provide an overview of the current state of the literature regarding patients' and caregivers' food allergy experiences and needs within the United States as well as a set of recommendations regarding how best to proceed with patient-centered development and evaluation of new food allergy treatments.

Recent findings The first pharmaceutical-grade product for peanut oral immunotherapy was approved in the United States for children aged 4-17 years following a successful international Phase 3 trial. This new treatment is only the first of several food allergy 
treatments currently under development. Patients will soon be presented with multiple options for food allergy treatment and will need to make decisions about what treatment is best for them.

Summary Allergy researchers and providers are encouraged to consider patients' perspectives and needs when developing and evaluating new food allergy treatments. Recommendations regarding next steps include the development of new patient-reported outcome tools, focus on psychosocial support, health disparities, and financial implications, and research harmonization and interdisciplinary collaboration.

Food allergy is a major public health concern in many countries around the world. In the United States (US), recent work suggests that it affects up to $8 \%$ of children and $10 \%$ of adults and is responsible for an emergency room admission every three minutes $[1 \bullet \bullet, 2-3]$. Historically, the only treatment for food allergy has been complete allergen avoidance combined with rescue medications used to ameliorate symptoms resulting from accidental exposures when they do occur. Thus, patients with food allergy and their families must adhere to multiple components of a food allergy management regimen in order to prevent allergic reactions, including reading food labels, preparing allergen-free meals, and advocating for safety when eating outside the home and when children are under the care of teachers and other care providers $[4,5]$. When anaphylaxis does occur, it must be treated immediately with epinephrine; therefore, patients and/or their caregivers must be prepared to treat allergic reactions at all times by carrying epinephrine auto-injectors and knowing when and how to administer them [4]. Complete adherence to an allergen avoidance diet is challenging and up to $40 \%$ of children with food allergy experience a severe allergic reaction [6-8]. Advances in food allergy research over the past decade have yielded immunotherapy treatments delivered by oral (OIT), epicutaneous (EPIT), and sublingual (SLIT) routes, as well as novel approaches in earlier-stage development. These advances have moved the field towards a future where new options for daily food allergy management may be available for many patients; indeed, the Food and Drug Administration (FDA) approved the first drug indicated for food allergy in January 2020. As groundbreaking as this new treatment and other treatments in the research pipeline are, their value is dependent on the extent to which they address food allergy patients' needs and how feasible it is to integrate them into families' daily lives. Importantly, there is no evidence that any food allergy therapy currently available or in ongoing research offers the chance of cure, which means that daily allergy management is still necessary and the psychosocial impact remains even during treatment. It is vital that researchers and allergy providers use patients' and caregivers' food allergy treatment preferences and experiences as a guiding beacon while developing and implementing new treatments. The purpose of this review is to provide 1) an overview of the current state of the literature regarding patients' and caregivers' food allergy experiences and needs and 2) a set of recommendations regarding how best to proceed with patient-centered development and evaluation of new food allergy treatments. This review is focused on developments within the United States. 


\section{The psychosocial impact of food allergy}

Over the past two decades, psychosocial research about food allergy families has led to a growing recognition that allergen avoidance and emergency preparedness alters the daily life of food allergy families $[9 \bullet \bullet, 10]$. Indeed, children with food allergy and caregivers report stress and anxiety comparable to other pediatric chronic illness populations [11]. Caregivers may experience fear about their child's safety after a first allergic reaction and uncertainty about how to adapt to a new way of life $[9 \bullet \bullet]$. Reading food labels and preparing allergen-free meals takes time and mental energy above and beyond typical meal planning, and requires families to rely on others to keep their child safe $[9 \bullet \bullet]$. Food labels can be confusing, as the current US law only requires 8 major allergens to be identified in "plain language" [12], precautionary allergen labeling in the US is not regulated, and labeling practices vary across countries; indeed, many families are confused about labeling and if/how it is regulated [13•]. Outside the home, asking about the ingredients in foods served at restaurants and social gatherings and making decisions about their safety requires self-confidence in one's ability to advocate for safety and a willingness to potentially go against social norms. Many families report fear about accidental allergen exposure. Although some degree of worry about allergic reactions is adaptive and promotes appropriate food allergy management, for some families, this anxiety is maladaptive and interferes with participation in appropriate academic and social activities $[9 \bullet \bullet, 11]$.

Since the early 2000s, much research regarding food allergy has focused on food allergy-related quality of life (QoL), or the individual's subjective, multidimensional assessment of their position in life (i.e., physical, psychological, and social) relative to their goals, expectations, and standards [14, 15]. A rigorously developed set of food allergy-specific QoL measures, the Food Allergy Quality of Life questionnaires (FAQLQ) [16] and the Food Allergy Quality of Life-Parental Burden questionnaire [17], have been widely used by researchers to assess the impact of food allergy management on children, caregivers, and adults with food allergy; however, these tools are not intended for use in routine clinical practice and were not developed to measure treatment effects. Collectively, there is evidence that food allergy can negatively impact multiple domains of QoL, including social and dietary limitations, feelings of difference from peers, and daily anxiety, and that some food allergy experiences may differentially affect QoL, such as anaphylaxis, number of food allergies and the specific allergens that need to be avoided, and participation in oral food challenges (OFC) [18].

Finding balanced integration of food allergy management into daily life is important to promoting healthy adjustment to life with food allergy [19]. This could be easily identified as an important goal of interventional therapy, that is, a useful treatment should normalize experience as much as possible and relieve families of some of the burdens and stressors imposed by living with food allergy. QoL is an important component of such an outcome, but is not the only one; new tools designed to measure treatment benefit are necessary and will be discussed further. As new treatments for food allergy are developed, it is imperative that these treatments improve the aspects of daily life that are reported by families to be stressful, while minimizing additional treatment burden. Keeping this balance in mind when developing new treatments will ensure the greatest positive impact on patients' well-being.

\section{Patient-reported priorities}

Until recently, the patient voice has not been systematically involved in efforts to prioritize research into the diagnosis, prevention, and management of food allergies, or in efforts to address disparities in care. To remedy this research gap, the world's largest patient advocacy group, Food Allergy Research \& Education (FARE), organized a multidisciplinary Outcomes Research Advisory Board (ORAB) composed of 44 key stakeholders, including food allergic patients, caregivers of food allergic children, clinicians, school nurses, educators, research scientists, and food allergy advocates. The ORAB focused on developing solutions that would improve the QoL of individuals with food allergies as well as 
those in the broader food allergy community. Results indicated five topics that stakeholders believed to be the most critical areas requiring additional research: improved diagnostic tools to predict reaction outcomes, development of therapeutic options to prevent the most severe allergy symptoms, implementation of global labeling language that simplifies allergen avoidance without excluding safe options, health literacy outreach to ensure that all patients and families have useful information, and support mechanisms to help remedy the profound psychosocial toll of food allergy [20].

Subsequently, the ORAB expanded the project scope, and undertook an indepth analysis of the availability and demand for mental health services for the food allergic community. Results highlighted the need to incorporate psychosocial evaluations into clinical trials, both to meet the needs of patients and families and to gather longitudinal data about how trial participation affects QoL [21•]. It also emphasized the importance of providing psychosocial support as part of comprehensive food allergy care and that food allergy clinics include this aspect of care in their fellowship training programs [21•].

\section{Current and future food allergy treatment options}

The highest-ranked patient-oriented research priority identified by the FARE ORAB was the development of new treatments to reduce the severity of food allergies. Allergy providers have long recognized the need to provide interventional treatment to patients with food allergy in an attempt to improve their condition. The first use of OIT in the literature was reported in 1908, and other anecdotal reports subsequently appeared but the practice was not widespread [22]. Renewed interest in therapeutic development emerged as food allergy increased in prevalence towards the end of the twentieth century, culminating in a landmark attempt in the 1990s to treat peanut allergy with subcutaneous injections of aqueous peanut extract [23]. This important work established that controlled exposure to food allergens could yield reduction in clinical sensitivity (an outcome called "desensitization"), but ultimately this approach was abandoned when the scientific community judged this approach to result in excessive toxicity [24]. As a result, in the early 2000s, researchers turned their attention back to the early reports of OIT and began to develop protocols to assess the safety and efficacy of this approach, establishing the precedent of an exit OFC to measure desensitization as a primary endpoint. Many OIT trials were undertaken around the world, studying a variety of methods to induce desensitization to common food allergens alone (typically peanut, milk, or egg, though other foods have also been studied) or, in a few studies, in combination. This seminal worldwide research effort in the 2000s-2010s has been reviewed extensively elsewhere, and a detailed summary is beyond the scope of this article. But this critical period in the field catalyzed two major developments in parallel. First, these initial trials, largely performed in academic medical centers, formed an evidence base that ultimately led to the commercial development and approval of a pharmaceutical-grade product for peanut OIT in US children aged 4-17 years following a successful international Phase 3 trial in which efficacy was measured with a double-blind, placebo-controlled food challenge $[25 \bullet \bullet]$. Simultaneously, a relatively small proportion of the US allergy practitioners began to offer OIT to patients using non-standardized 
commercial food preparations and individualized protocols of their own design. Similar trends occurred in Europe, where recent guidelines have endorsed the use of OIT in certain settings [26]. As a result, families in the US increasingly face an important and unprecedented decisional dilemma: to continue with allergen avoidance or pursue treatment, either with "non-standardized," or in the case of peanut allergy, FDA-approved forms of OIT. This dilemma may soon grow more complex for families with school-aged peanutallergic children, if Viaskin EPIT is eventually approved for use. Although at the time of publication there remains uncertainty about the likelihood of approval, parents of children aged 4-11 years could potentially have a choice of four different approaches to manage their child's peanut allergy. Ongoing early-stage research programs may one day yield new technologies in the treatment of peanut allergy (three examples include: chemically-modified allergens; fully synthetic peptides; and DNA vaccines, all delivered by injection), further increasing complexity. Meanwhile an ongoing Phase 3 trial testing omalizumab as monotherapy, and in conjunction with OIT, in the treatment of peanut allergy plus at least two other allergies (e.g., milk, egg, wheat, cashew, walnut, and hazelnut) could have a transformational impact on food allergy practice by tackling several food allergies simultaneously. Such strategies are urgently needed for families navigating the challenges of multiple food allergy, and in recognition of this unmet need, omalizumab was granted Fast-Track status for food allergy indication by FDA.

As a result of these research advances, the clinical practice of allergy is at a major inflection point. Treatment options are already widely available in peanut allergy and will very likely continue to increase for other age groups, other foods, and with other approaches. Despite this progress, major gaps remain in our understanding of how best to select patients for each therapy, how to optimize treatment regimens to enhance outcomes, and how to ensure that the treatment benefit in terms of overall well-being is directly measured, rather than assumed, based on an OFC outcome.

\section{Patients' expectations for and experiences with new food allergy treatments}

Novel treatments have the potential to positively impact the lives of patients with food allergy and their caregivers by reducing the risk for allergic reaction in response to accidental allergen exposure and concomitantly reducing fear related to it $[27,28]$. However, the new food allergy treatments that have been developed to date will be long-term, non-curative treatments that require clinical monitoring beyond current allergy practice due to the daily possibility of allergic reactions, including anaphylaxis, and will still require patients to avoid allergens and carry epinephrine auto-injectors [25••, 29-31]. Families may experience stress and anxiety related to daily doses and experience adherence challenges throughout treatment. 
Early research in the US regarding the impact of clinical trial-based single food OIT and EPIT is sparse, with the preponderance of psychosocial research about novel treatments found outside the US and/or conducted with samples undergoing individualized OIT protocols in private practice. That being said, initial research on food allergy-related QoL and treatment satisfaction related to OIT notes a positive psychosocial impact due to increased dietary options and social inclusion and decreased anxiety about allergic reactions, but some patients report anxiety about daily doses and food aversions [32]. Regarding EPIT, a small initial study indicated that most participants' food allergy-related QoL improved one year after initiating treatment [33]. In a larger-scale longitudinal EPIT study, participants in clinical trials who reached the primary endpoint exhibited improved QoL across multiple psychosocial domains at 24 months post-enrollment compared to patients in the placebo group [34•]. Thus, there appears to be some positive psychosocial impact of OIT and EPIT on patients and families, but additional research is needed to further understand what aspects of immunotherapy contribute to QoL improvements. For example, Duca and colleagues [35•] conducted a cost-benefit analysis of food immunotherapy and raised important questions regarding the mechanism by which immunotherapy positively impacts QoL: is all improvement front-loaded by the information gained from an OFC prior to clinical trial participation, is it due to increased contact with the allergy team which may increase food allergy knowledge and self-efficacy, is it due to desensitization, or is it a combination of these factors? The actual impact on food allergy-related QoL is unclear and is likely to be dependent on both families' pre-existing food allergy-related QoL while following an avoidance diet and their expectations for the ways in which QoL will change.

A recent Institute for Clinical and Economic Review (ICER) report regarding OIT and EPIT for peanut allergy indicated that QoL improvements are likely to be one of the primary benefits of immunotherapy [36•]; thus, families whose children are eligible for immunotherapy must individually assess the cost and benefits to both avoidance and immunotherapy treatment approaches within the context of their family's desires, needs, and values, and determine which treatment will have the greatest positive impact for them [37]. Research regarding OFCs suggests that patients' reasons for pursuing novel food allergy treatment and experiences during treatment may be affected by caregiver-allergist rapport, the time commitment for participation, the value placed on eating the food, treatment success, and anxiety about allergic reactions [38-40]. The highly individual nature of this decision is highlighted by the extant literature about parents' reasons for pursuing OIT and EPIT. Many caregivers who express interest in or enroll in clinical trials for immunotherapy self-report that they fear fatal anaphylaxis and want protection from accidental allergen exposure, but other caregivers choose this treatment because they want their children to be able to eat allergens freely $[27,28]$. The importance of informing families during the consent process about what they can expect during and at the end of treatment cannot be overstated, as incomplete information and/or unmet treatment expectations may have implications for adherence (which may lead to serious medical and/or psychological 
adverse events), treatment satisfaction, and the families' trust in the medical community. There is a growing call for family-provider collaboration when making treatment decisions (i.e., shared decision-making) [36•, 41, 42 ], which will require providers to spend additional time with patients, have a robust understanding of the risks/benefits of new treatments, and be sensitive to the unique needs and expectations of each patient and family.

\section{Recommendations for allergy researchers and providers}

Two decades of research demonstrate that food allergy greatly impacts the psychosocial functioning of affected patients and caregivers. Recent advances in food allergy research have led to a new era in food allergy treatment, one which may give patients more choices about how they manage food allergy. Patients' perspectives need to be central in the development of new treatments. Recommendations for allergy researchers and providers who strive to develop and evaluate patient-centered food allergy treatments fall into four categories: 1) new tools, 2) psychosocial support, 3) health disparities and financial implications, and 4) research harmonization and interdisciplinary collaboration.

The extant patient-reported outcomes (PRO) used to evaluate novel treatments are limited and likely not capturing patients' perceived treatment value or burden, or the true impact of these treatments on QoL. To date, the only psychosocial measures included in clinical trial research are QoL measures, which do not account for other important PROs involving decisional conflict, anxiety, stress, adherence concerns associated with treatment, suffering related to adverse events, or treatment satisfaction. Furthermore, previous studies have not directly assessed developmental challenges, yet child age is likely to significantly impact treatment decision-making and the challenges that arise (e.g., dose refusal and planning and execution of daily doses), and treatment challenges are likely to change as children age (e.g., older children have agency in medical decision-making). The FAQLQ measures, although valid and reliable for patients utilizing an avoidance diet, are insufficient to capture the nuances of the lived experience of daily medication doses and their concomitant increase in risk for allergic reaction and were not developed for this purpose. For example, if families desire a decrease in anxiety related to accidental allergen exposure, measures of food allergy-specific anxiety are needed in order to capture change over the course of treatment [43•]. Conversely, emerging research suggests that anaphylaxis may be associated with acute trauma symptoms; thus, development of tools that assess the immediate and long-term impact of allergic reactions, including anaphylaxis due to daily treatment doses, is also warranted [44-46]. Furthermore, research to elucidate what patients want and how novel treatments address them are needed, but this alone is not enough; we 
Psychosocial support

also need to understand what patients are willing to "spend" to get them, both with respect to financial cost and time/emotional cost. A perfect food allergy treatment will not be utilized by the public if it demands too much from patients and families in exchange. New tools must be designed for longitudinal use in routine practice to measure multiple PROs beyond QoL and to assess how they are impacted by interventional treatments. This will take years of focused research effort and is critical to understand the true value of each treatment and lay the groundwork for the comparative effectiveness research needed to evaluate treatments head-to-head.

Successful implementation and adoption of novel food allergy treatments will require allergy health care providers to be thoughtful about their clinics' abilities to integrate complex, time-consuming treatment into current allergy clinics in a manner that is also sensitive to their patients' needs. The existing novel treatments require daily adherence to medication, which is not a treatment model to which food allergy patients are accustomed. Successful adherence to these novel treatments is most likely when the behavioral health aspects of the treatment are addressed. We need to carefully examine allergy health care providers' and patients' and parents' thoughts and experiences with these treatments in order to develop allergy clinical programs that support them and evidence-based psychosocial interventions. Not only is this crucial for patient and family adjustment to treatment, but there is potential for severe allergic reactions if adherence is poor; thus, the behavioral aspects of treatment are of paramount importance. That being said, there are far too few mental health professionals with expertise in food allergy in the US to provide care to patients and families who need it $[21 \bullet]$; training programs for mental health professionals are needed and allergy clinics are encouraged to think about ways to develop multidisciplinary teams that include mental health professionals.

Health disparities and financial implications

Knowing that food allergies cost families in the US $\$ 24.8$ billion annually [47], and that disparities in food allergy costs by race and socioeconomic strata exist, the ORAB prioritized health disparities as unaddressed needs in the field [48]. African-American children were reported to spend less on direct medical and out-of-pocket costs compared to other racial groups [48]. Children from the lowest household income stratum incurred 2.5 times the cost that children from the highest stratum spent on emergency department and hospitalization costs [48]. It is possible that children from lower-income families may be at higher risk for accidental ingestion and anaphylaxis because of barriers such as limited access to specialty care and allergen-free foods, as well as epinephrine auto-injectors. 
Lack of access to allergists may also inhibit knowledge of and access to food allergy treatments. Recent work confirms that disparities in OIT knowledge exists by socioeconomic status and that further outreach initiatives are needed to disseminate information and provide equitable access to OIT [49•]. Furthermore, the amount of time (both office visits and at home) that is required to participate in new treatments can be substantial, which will make treatment unrealistic for families who have fewer resources and/or less employment flexibility. Indeed, in a study about participation in OFCs, concerns about time were a decision-making factor for families [38]. Although some work has been accomplished to raise awareness of these disparities, much more work needs to be completed to address the economic and knowledge disparities and inequalities in food allergy.

Food allergy is a growing global public health concern [50]. Many research teams around the world are actively pursuing new treatment options and large-scale international clinical trials are increasingly common (e.g., the PALISADE study regarding OIT[25] and the PEPITES study regarding EPIT [51]). Effort should be made to continue to harmonize and align research among research teams, standardize the collection and reporting of outcome variables [52], and share clinical trial data in order to increase the pace of discovery and avoid redundancy, which will ultimately be better for patients. Indeed, there has been a call to share data from clinical trials by the Institute of Medicine and other groups [53, 52]. Consistent language about the risks, benefits, and alternatives to treatment is also needed.

Additionally, there is an urgent need to include new disciplines in food allergy research in order to ensure that all aspects of health are accounted for, not just the biological mechanisms of food allergy, and that the treatments under development can be realistically implemented across a global patient population. For example, behavioral researchers can offer insight regarding patient behavior, based on theoretical models such as the Health Belief Model [54], which may help medical researchers develop treatments that are most likely to be utilized effectively by patients. Additionally, despite food allergies affecting $8-10 \%$ of the population, public health researchers are currently minimally involved with food allergy research, yet have insight regarding health disparities that are vital to the development of equitable food allergy treatment. A call to action from the National Academies of Medicine highlighted the urgent need for enhanced involvement in food allergy research from leading public health agencies such as the Centers for Disease Control [52]. A recent report regarding the impact of COVID-19 on food insecure allergic families provided public policy recommendations that would positively impact families across the socioeconomic spectrum; although not directly related to novel food allergy treatments, this 
paper exemplifies the type of cross-disciplinary collaboration that will ensure the allergy community is sensitive to the needs of all food allergy families [55].

\section{Conclusion}

A new era in food allergy has arrived. Interventional treatments are now available to some families in routine practice, including the first drug product to be approved by the FDA to treat food allergy. Over time, an increasing number and type of therapeutic options are expected to become widely available through further research and development. Although these achievements represent a significant paradigm shift, the successful translation of therapies into real-world practice will require a better understanding of patient-centered outcomes, an area of food allergy research that has been neglected to date. We present here our impressions of the remaining gaps and call for increased attention to these critically important research needs. Only when patient preferences and expectations are taken into account will the promise of therapeutic development become fully realized.

\section{References and Recommended Reading}

Papers of particular interest, published recently, have been highlighted as:

- Of importance

- Of major importance

1.• Gupta RS, et al. The public health impact of parentreported childhood food allergies in the United States. Pediatrics. 2018;142(6):e2018123.

Pivotal paper regarding the prevalance of food allergy in the United States.

2. Sicherer SH, Muñoz-Furlong A, Godbold JH, Sampson HA. US prevalence of self-reported peanut, tree nut, and sesame allergy: 11-year follow-up. J Allergy Clin Immunol. 2010;125(6):1322-6.

3. Gupta RS, Warren CM, Smith BM, Jiang J, Blumenstock JA, Davis MM, et al. Prevalence and severity of food allergies among US adults. JAMA Netw Open. 2019;2(1):e185630.

4. Yawn P. Fenton summary of the NIAID-sponsored food allergy guidelines. Am Fam Physician. 2012;86(1):43-50.

5. Herbert L, Shemesh E, Bender B. Clinical management of psychosocial concerns related to food allergy. J Allergy Clin Immunol Pract. 2016;4(2):205-13.

6. Gupta RS, Springston EE, Warrier MR, Smith B, Kumar R, Pongracic J, et al. The prevalence, severity, and distribution of childhood food allergy in the United States. Pediatrics.

2011;128(1):e9-17.

7. Ross MP, Ferguson M, Street D, Klontz K, Schroeder T, Luccioli S. Analysis of food-allergic and anaphylactic events in the National Electronic Injury Surveillance System. J Allergy Clin Immunol. 2008;121(1):166-71.

8. Boyce JA, et al. Guidelines for the diagnosis and management of food allergy in the United States: report of the NIAID-sponsored expert panel. J Allergy Clin Immunol. 2010;126(6 Suppl):S1-58.

9.• Moen OL, Opheim E, Trollvik A. Parents experiences raising a child with food allergy: a qualitative review. J Pediatr Nurs. 2019;46:e52-6.

Meta-synthesis of qualitative research regarding parents of children with food allergy.

10. Johnson SF, Woodgate RL. Qualitative research in teen experiences living with food-induced anaphylaxis: a meta-aggregation. J Adv Nurs. 2017;73(11):2534-46. 
11. Polloni L, Muraro A. Anxiety and food allergy: a review of the last two decades. Clin Exp Allergy. 2020;50(4):420-41.

12. Food and Drug Administration. Food Allergen Labeling and Consumer Protection Act of 2004 (Public Law 108-282, Title II). 2004. http://www.fda.gov/Food/ GuidanceRegulation/

GuidanceDocumentsRegulatoryInformation/ Allergens/ucm106187.htm. Accessed 15 June 2020.

13. Marchisotto MJ, et al. Food allergen labeling and purchasing habits in the United States and Canada. J Allergy Clin Immunol Pract. 2017;5(2):345-3.

This paper reviews the difficulty that food allergy consumers have when selecting safe foods.

14. WHOQOL. The World Health Organization Quality of Life assessment (WHOQOL): position paper from the World Health Organization. Soc Sci Med.

1995;41(10):1403-9.

15. Sicherer SH, Noone SA, Munoz-Furlong A. The impact of childhood food allergy on quality of life. Ann Allergy Asthma Immunol. 2001;87(6):461-4.

16. DunnGalvin A, de BlokFlokstra BMJ, Burks AW, Dubois AEJ, Hourihane JO'B. Food allergy quality of life questionnaire for children aged 0-12 years: content, construct, and cross-cultural validity. Clin Exp Allergy. 2008;38(6):977-86.

17. Cohen BL, Noone S, Muñoz-Furlong A, Sicherer SH. Development of a questionnaire to measure quality of life in families with a child with food allergy. J Allergy Clin Immunol. 2004;114(5):1159-63.

18. Kansen HM, le TM, Meijer Y, Flokstra-de Blok BMJ, Welsing PMJ, van der Ent CK, et al. The impact of oral food challenges for food allergy on quality of life: a systematic review. Pediatr Allergy Immunol. 2018;29(5):527-37.

19. Klinnert MD, McQuaid EL, Fedele DA, Faino A, Strand M, Robinson J, et al. Children's food allergies: development of the food allergy management and adaptation scale. J Pediatr Psychol. 2015;40(6):572-80.

20. Bilaver LA, Sharma HP, Gupta RS, Herbert LJ, Marchisotto MJ, Aktas ON, et al. Food allergy research priorities: results from a patient-centered study. J Allergy Clin Immunol Pract. 2019;7(7):2431-3.

21. Herbert L, et al. Availability of mental health services for patients with food allergy. J Allergy Clin Immunol In Pract. 2019;7(8):2904-.

This paper documents the lack of mental health services for patients with food allergy.

22. Gillespie EH. A case of food poisoning in man probably caused by the consumption of a duck's egg (further report). Mon Bull Minist Health Emerg Public Health Lab Serv. 1946;5:157.

23. Oppenheimer JJ, et al. Treatment of peanut allergy with rush immunotherapy. J Allergy Clin Immunol. 1992;90(2):256-62.

24. Nelson HS, et al. Treatment of anaphylactic sensitivity to peanuts by immunotherapy with injections of aqueous peanut extract. J Allergy Clin Immunol. 1997;99(6 Pt 1):744-51.
25.• Vickery B, et al. AR101 Oral Immunotherapy for Peanut Allergy. N Engl J Med. 2018;379(21):1991-200.

Pivotal paper detailing the results of the AR101 clinical trial.

26. Pajno GB, Fernandez-Rivas M, Arasi S, Roberts G, Akdis $\mathrm{CA}$, Alvaro-Lozano M, et al. EAACI Guidelines on allergen immunotherapy: IgE-mediated food allergy. Allergy. 2018;73(4):799-815.

27. Greenhawt M, Marsh R, Gilbert H, Sicherer S, DunnGalvin A, Matlock D. Understanding caregiver goals, benefits, and acceptable risks of peanut allergy therapies. Ann Allergy Asthma Immunol. 2018;121(5):575-9.

28. Kao LM, Greenhawt MJ, Warren CM, Siracusa M, Smith BM, Gupta RS. Parental and parent-perceived child interest in clinical trials for food allergen immunotherapy. Ann Allergy Asthma Immunol. 2018;120(3):331-333.e1.

29. Nurmatov U, Dhami S, Arasi S, Pajno GB, FernandezRivas M, Muraro A, et al. Allergen immunotherapy for IgE-mediated food allergy: a systematic review and meta-analysis. Allergy. 2017;72(8):1133-47.

30. Varshney P, Steele PH, Vickery BP, Bird JA, Thyagarajan A, Scurlock AM, et al. Adverse reactions during peanut oral immunotherapy home dosing. J Allergy Clin Immunol. 2009;124(6):1351-2.

31. AImmune Therapuetics. Highlights of Palforiza Prescribing Information. https://www.palforzia.com/ static/pi_palforzia.pdf. Accessed 15 June 2020.

32. LeBovidge J, et al. The psychological impact of oral immuntherapy for children with food allergy: perceived benefits and treatment burden. Clin Pract Pediatr Psychol. 2014;2:13-26.

33. Lewis MO, Brown-Whitehorn TF, Cianferoni A, Rooney C, Spergel JM. Peanut-allergic patient experiences after epicutaneous immunotherapy: peanut consumption and impact on quality of life. Ann Allergy Asthma Immunol. 2019;123(1):101-3.

34. Greenhawt M, et al. Improvements in quality of life following epicutaneous peanut immunotherapy: PEPITES and PEOPLE studies. Ann Allergy Asthma Immunol. 2019;123(5):S16-.

This paper documents quality of life improvements related to EPIT.

35. Duca B, Patel N, Turner PJ. GRADE-ing the benefit/risk equation in food immunotherapy. Curr Allergy Asthma Rep. 2019;19(6):3.

This paper reviews the benefits and risks of food immunotherapy.

36. Institute for Clinical and Economic Review. Oral Immunotherapy and Viaskin Peanut for Peanut Allergy: Effectiveness and Value. California Technology Assessment Forum. 2019; Thorough review of the value of oral immunotherapy and epicutaneous immunotherapy for peanut allergy.

37. Dufresne E, Poder TG, Begin P. The value of oral immunotherapy. Allergy. 2020;75(6):1291-3.

38. Herbert LJ, Dahlquist LM, Bollinger ME. Maternal intolerance of uncertainty, anxiety, and adherence with 
food challenge referrals. J Health Psychol.

2013;18(9):1209-19.

39. Davis N, Egan M, Sicherer SH. Factors resulting in deferral of diagnostic oral food challenges. J Allergy Clin Immunol Pract. 2015;3(5):811-2.

40. Eigenmann PA, Caubet JC, Zamora SA. Continuing food-avoidance diets after negative food challenges. Pediatr Allergy Immunol. 2006;17(8):601-5.

41. Anagnostou A, Hourihane JO, Greenhawt M. The role of shared decision making in pediatric food allergy management. J Allergy Clin Immunol Pract. 2020;8(1):46-51.

42. Greenhawt M. Shared decision-making in the care of a patient with food allergy. Ann Allergy Asthma Immunol. 2020;125(3):262-7.

43. Dunlop JH, Keet CA. Goals and motivations of families pursuing oral immunotherapy for food allergy. J Allergy Clin Immunol Pract. 2019;7(2):662-3.

44. Weiss D, Marsac ML. Coping and posttraumatic stress symptoms in children with food allergies. Ann Allergy Asthma Immunol. 2016;117(5):561-2.

45. Chung MC, Walsh A, Dennis I. Trauma exposure characteristics, past traumatic life events, coping strategies, posttraumatic stress disorder, and psychiatric comorbidity among people with anaphylactic shock experience. Compr Psychiatry. 2011;52(4):394-404.

46. Polloni L, et al. Post-anaphylaxis acute stress symptoms: a preliminary study on children with foodinduced anaphylaxis and their parents. J Allergy Clin Immunol In Pract. 2020. https://doi.org/10.1016/j. jaip.2020.06.036 Online ahead of print.

47. Gupta R, Holdford D, Bilaver L, Dyer A, Holl JL, Meltzer D. The economic impact of childhood food allergy in the United States. JAMA Pediatr. 2013;167(11):1026-31.

48. Bilaver LA, Kester KM, Smith BM, Gupta RS. Socioeconomic disparities in the economic impact of childhood food allergy. Pediatrics. 2016;137(5):e20153678.

49. $\quad$ Roach A, et al. Awareness of oral immunotherapy (OIT) among a nationally-representative sample of food allergy patients/caregivers is critically lacking. J Allergy Clin Immunol. 2020;145(2):AB34.

This paper highlights the lack of awareness among food alleryg patients regarding oral immunotherapy.

50. Loh W, Tang MLK. The epidemiology of food allergy in the global context. Int J Environ Res Public Health. 2018;15(9):2043.

51. Fleischer DM, Greenhawt M, Sussman G, Bégin P, Nowak-Wegrzyn A, Petroni D, et al. Effect of epicutaneous immunotherapy vs placebo on reaction to peanut protein ingestion among children with peanut allergy: the PEPITES randomized clinical trial. JAMA. 2019;321(10):946-55.

52. National Academies of Sciences, Engineering, and Medicine. Finding a path to safety in food allergy: assessment of the global burden, causes, prevention, management, and Public policy. Washington, DC: The National Academies Press; 2017.

53. Institute of Medicine. Sharing clinical trial data: Maximizing benefits, minimizing risk. Washington, DC: The National Academy Press; 2015.

54. Jones CJ, Smith HE, Frew AJ, Toit GD, Mukhopadhyay $S$, Llewellyn CD. Explaining adherence to self-care behaviours amongst adolescents with food allergy: a comparison of the health belief model and the common sense self-regulation model. British J Health Psychol. 2014;19(1):65-82.

55. Brown E, Das R, Brewer AG, Martinez E, Bilaver LA, Gupta RS. Food insecure and allergic in a pandemic: a vulnerable population. J Allergy Clin Immunol In Pract. 2020;8(7):2149-51.

\section{Publisher's Note}

Springer Nature remains neutral with regard to jurisdictional claims in published maps and institutional affiliations. 